\title{
Optical metamaterials with different metals
}

\author{
Nian-Hai Shen,, , 田 Thomas Koschny, ${ }^{1,2}$ Maria Kafesaki, ${ }^{2,3}$ and Costas M. Soukoulis ${ }^{1,2, \text {, }}$ \\ ${ }^{1}$ Ames Laboratory and Department of Physics and Astronomy, \\ Iowa State University, Ames, Iowa 50011, U.S.A. \\ ${ }^{2}$ Institute of Electronic Structure and Laser, FORTH, 71110 Heraklion, Crete, Greece \\ ${ }^{3}$ Department of Materials Science and Technology, \\ University of Crete, 71003 Heraklion, Crete, Greece
}

\begin{abstract}
We investigate the influence of different metals on the electromagnetic response of fishnet metamaterials in the optical regime. We found, instead of using a Drude model, metals with a dielectric function from experimentally-measured data should be applied to correctly predict the behavior of optical metamaterials. Through comparison of the performance for fishnet metamaterials made with different metals, i.e., gold, copper, and silver, we found silver is the best choice for the metallic parts compared to other metals, because silver allows for the strongest negative-permeability resonance and, hence, for optical fishnet metamaterials with a high figure-of-merit. Our study offers a valuable reference in the designs for optical metamaterials with optimized properties.

PACS numbers: 78.67.Pt, 78.20.-e, 42.25.Bs, 78.66.Bz
\end{abstract}

Research on metamaterials (MMs), i.e., artificial structures composed of tailored subwavelength building blocks, has been a burgeoning field in the past decade, due to great interest in both theoretical studies and practical applications,$\frac{1-5}{-5}$ MMs are found able to greatly improve our capabilities to manipulate electromagnetic radiation almost throughout the entire spectrum, providing many intriguing properties and phenomena, such as negative refractive index $n \stackrel{6.7}{=}$ superlensing, $\stackrel{-10}{=}$ and invisibility $\underline{\underline{11}-15}$ Following the efforts of pushing MMs to work in the optical regime $\underline{16}-18$ from the microwave regime,,$\frac{6.19}{,}$ intrinsic loss of constituents, especially metallic inclusions, has become a more severe problem. In the course of realizing negative $n$ in the visible regime, fishnet, i.e., a perforated metal-dielectric-metal sandwich, has substituted split-ring-resonator (SRR), which fails to provide negative $\mu$ above infrared frequencies $\stackrel{20}{\underline{2}}$ to become the most promising structure. Concerning strategies for reducing loss in a MM system, an appropriate selection of low loss materials as constituents of MMs is quite straightforward. The introduction of gain materials is believed to be the most efficient but also very challenging, 21,22 For pure passive structures, lower losses have been reported by building a stronglycoupled system $\underline{16,23}$ and by geometric optimizations $\stackrel{24}{\underline{2 n}}$ In the meantime, because of costly fabrication of MMs in the optical regime, guidance from simulations is helpful and desirable. However, it is noted all the related materials should be correctly modeled to accurately predict the behaviors of MMs. Here, we focus on the influence of metals in the electromagnetic response of MMs, show how we should model metals correctly, and which metal is the best choice for achieving low-loss negative-index MMs in the optical regime.

The discussions in this paper are divided into two parts: First, taking gold as an example, we compare the experimentally-measured Johnson-Christy (JC) data 25 with some Drude models in the optical regime and investigate their influence on the response of a fishnet MM.
Second, we study the performances of a MM in the visible regime based on different metals to show the best option of metal as a constituent of MMs to obtain a satisfying negative- $n$ property.

For noble metals, there are various sources providing different sets of frequency-dependent material data to describe them $\stackrel{25.26}{2}$ Because of the additional scattering for electrons resulting from the metal surfaces, material property of a thin-film metal is different from that of a bulk metal. JC data, specifically measured for thin film metals and proven independent of thickness between $25-50 \mathrm{~nm}$, is very appropriate for application in the modeling of MMs in the optical regime, in which the thickness of metallic inclusions is generally tens of nanometers. On the other hand, the Drude model, i.e., $\varepsilon(f)=\varepsilon_{\infty}-f_{p}^{2} /\left[f\left(f+i f_{c}\right)\right]$, where $f$ is the frequency, $\varepsilon_{\infty}$ is the offset value of permittivity, while $f_{p}$ and $f_{c}$ are plasma and collision frequencies, respectively, is also widely applied to describe metals. However, we must be careful that when our interested frequency band goes into the optical regime, the Drude model may no longer be valid, as shown below.

A widely-used Drude model (model 1$)^{27}$ in the field of MMs for gold has $\varepsilon_{\infty}=1, f_{p}=2175 \mathrm{THz}$, and $f_{c}=10.725 \mathrm{THz}$, with $f_{c}$ three times larger than the normal value for bulk metal shown in Ref. [24]. We show Drude model 1 with the frequency-dependent dielectric constant from 150 to $750 \mathrm{THz}$ compared to JC data in Fig. 1. We clearly see the discrepancy between the two for both real and imaginary parts of permittivity throughout the range. The experimental JC data include higher $\operatorname{Im}[\varepsilon]$, thereby a higher intrinsic loss in gold. Intuitively, the application of Drude model 1 for gold in the design of MMs, such as fishnet structures (see Fig. 2), may lead to an inaccurate and overly optimistic prediction of performance. On the other hand, we have searched for an improved Drude model for gold, which fits JC data better and found with $\varepsilon_{\infty}=9.6, f_{p}=2184$ $\mathrm{THz}$, and $f_{c}=17 \mathrm{THz}$, i.e., Drude model 2, agrees with 




FIG. 1: (Color online) Comparison of permittivity (both real and imaginary parts) for gold with Johnson-Christy data (symbols), Drude model 1 (blue lines), and Drude model 2 (black lines).

JC data perfectly up to $450 \mathrm{THz}$ (shown in Fig. 1). However, thereafter, the goodness of fit between Drude model 2 and JC data starts breaking for $\operatorname{Im}[\varepsilon]$. Therefore, for frequencies above $450 \mathrm{THz}$, even Drude model 2 will not be valid to describe gold's properties.

Provided the fishnet structure (shown in Fig. 2) is the most popular MM in the optical regime, due to the ease in fabrication and satisfying performance in achieving double negative $(\varepsilon<0$ and $\mu<0)$ property, we would like to take the fishnet as an example to show the influence on the electromagnetic response of MMs, when we apply different sets of material data for metal (gold) inclusions.

The first specific fishnet structure, geometric parameters presented in the caption of Fig. 3, has the expected interesting negative-index property within telecommunications wavelengths (around $1.5 \mu \mathrm{m}$ ). For the metal inclusions (gold), we apply, respectively, JC data, Drude models 1 and 2. On the other hand, the dielectric spacer is lossless magnesium oxide $(\mathrm{MgO})$ with $n=1.7$. We correspondingly show calculated reflection/transmission/absorption (RTA) information $\underline{28}$ and retrieved effective electromagnetic parameters, 29232 i.e., $n$, permittivity $(\varepsilon)$, and permeability $(\mu)$ in columns (a), (b), and (c), respectively. It should be pointed out that the retrieval process is not trivial in general, especially when metamaterials are anisotropic or bi-anisotropic 33 and EM wave is oblique incident $\underline{34}$ From Fig. 3, we find that even though all three cases show us double-negative properties, the results of Case (b) are moderately superior over the other two cases, i.e., more promising negative value of $\mu$ and figure-of-merit $(\mathrm{FOM}=|\operatorname{Re}[n] / \operatorname{Im}[n]|)$ are reached in Fig. 3(b), which result from a stronger magnetic resonance under the lower-loss consideration in Drude model 1. In comparison, Cases (a) and (c) present almost the same results, which are expected because of the perfect fit of Drude model 2 to JC data for gold within our present studied regime (see Fig. 1).



FIG. 2: (Color online) Sketch of the fishnet structure: $a_{x}$ and $a_{y}$ are the unit cell sizes along $x$ and $y$ directions, respectively. $w_{x}$ and $w_{y}$ show the hole size. $t_{m}$ and $t_{d}$ are the thicknesses of metallic cladding and dielectric spacer, respectively.

In Fig. 4, we show simulated RTA results and retrieved spectra of $n, \varepsilon$, and $\mu$ for the fishnet structure 2, specifically designed to work in the visible regime. Again, columns (a), (b), and (c) correspond to the cases with gold modeled by JC data, Drude models 1 and 2, respectively. From Fig. 4(a), a magnetic resonance occurs around $450 \mathrm{THz}$ with positive values of $\mu$ throughout the region. Nevertheless, negative $n$ does exist with quite low values of FOM ( 0.5 at maximum). Because of the discrepancy for $\operatorname{Im}[\varepsilon]$ of gold between Drude model 2 and JC data above $450 \mathrm{THz}$, the electromagnetic response of the fishnet shown in Fig. 4(c) is significantly different from that in Fig. 4(a) for high frequencies. In comparison, Fig. 4(b) still shows simultaneously negative $\varepsilon$ and $\mu$, which is nonrealistic. It is determined magnetic resonance occurs at a higher frequency compared to that in the other two cases.

Therefore, we obtain the first important conclusion: to correctly predict the electromagnetic behavior of MMs in the optical regime (both telecommunication and visible wavelengths), the metallic inclusions should be modeled with accurate data, like experimental JC data. Drude model 1 , though widely used, is inaccurate or even invalid to describe gold in the optical regime and may deceive us to show the promising negative $\mu$, which is nonrealistic for related MMs. An improved Drude model 2 fits JC data perfectly up to $450 \mathrm{THz}$ and provides an alternate for modeling gold, when discrete JC data cannot be applied, such as in the time domain calculations ${ }^{35}$

In the following section, we would like to study the influence of different metals to a metallodielectric MM in the visible regime to show which metal will be the best 



FIG. 3: (Color online) Simulated reflection, transmission, absorption, and retrieved electromagnetic parameters for the fishnet shown in the inset of Fig. 2 with $a_{x}=500 \mathrm{~nm}, a_{y}=600 \mathrm{~nm}, w_{x}=200 \mathrm{~nm}, w_{y}=350 \mathrm{~nm}, t_{m}=30 \mathrm{~nm}$, and $t_{d}=40 \mathrm{~nm}$ for cases in which metal (gold) is modeled by discrete JC data (a), Drude model 1 (b), and Drude model 2 (c).


FIG. 4: (Color online) Simulated reflection, transmission, absorption, and retrieved electromagnetic parameters for the fishnet shown in Fig. 2 with $a_{x}=200 \mathrm{~nm}, a_{y}=220 \mathrm{~nm}, w_{x}=100 \mathrm{~nm}, w_{y}=120 \mathrm{~nm}, t_{m}=30 \mathrm{~nm}$, and $t_{d}=40 \mathrm{~nm}$ for cases in which metal (gold) is modeled by discrete JC data (a), Drude model 1 (b), and Drude model 2 (c). 


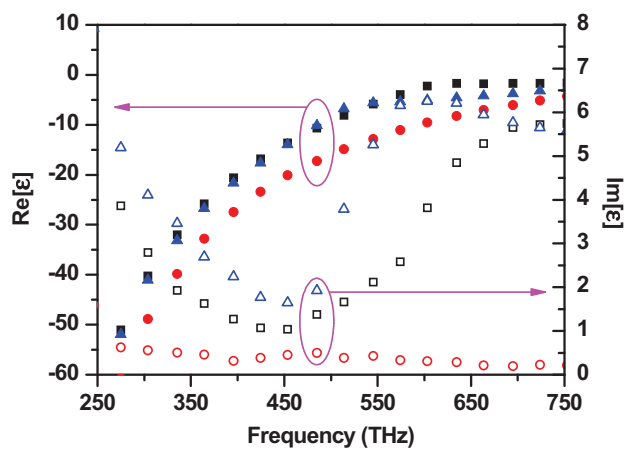

FIG. 5: (Color online) Comparison of real (solid symbols) and imaginary (hollow symbols) parts of permittivities for gold (square), silver (circle), and copper (triangle).
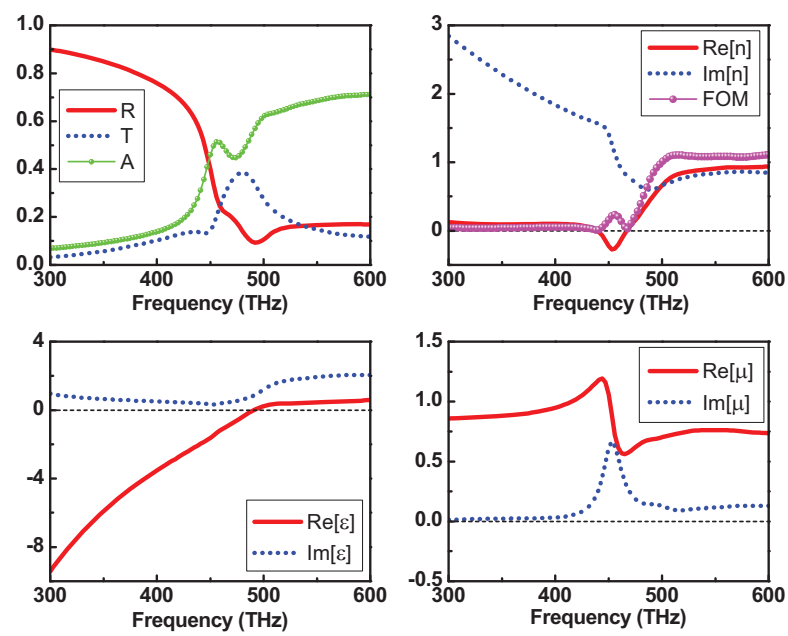

FIG. 6: (Color online) Simulated reflection, transmission, absorption, and retrieved electromagnetic parameters for structure 2 , in which, copper metal is applied with the corresponding JC data.

choice to benefit MMs in the achievement of a negativeindex property with a high FOM. Based on our above discussions, to study the properties of MMs in the optical regime accurately, experimentally-measured material data are always preferred to apply to the metal inclusions. Figure 5 shows JC data, i.e., both real and imaginary parts of permittivity from 250 to $750 \mathrm{THz}$, for thin film of gold, copper, and silver. As seen in Fig. 5 , throughout this frequency range, silver has the lowest loss, so a silver-made fishnet might be able to render the most promising property. However, such a speculation needs to be confirmed through the comparison of the real performances for a MM made with different metals. Using fishnet structure 2, whose geometric parameters are shown in the caption of Fig. 4, as an example, we perform simulations obtaining RTA information and then retrieve the effective $n, \varepsilon$, and $\mu$ of the structure.

The case for gold metal in the fishnet has been pre- sented in Fig. 4(a): a magnetic resonance occurs at around $450 \mathrm{THz}$ without any negative $\mu$, but negative $n$ is achieved with a quite low FOM ( $\sim 0.5$ at maximum). In comparison, the cases for copper- and silver-made fishnet are shown, respectively, in Figs. (6) and (7). With copper, we find the performance of the fishnet is even worse than the case with gold, i.e, FOM ( $\sim 0.25$ at maximum) is very low within the narrow band corresponding to negative $n$. The results for the case with silver are really exciting: Simultaneously negative $\varepsilon$ and $\mu$ are observed in a narrow band around $500 \mathrm{THz}$, leading to a negative $n$ with quite satisfying FOM (more than 2 at maximum). Note here we also tried the case with aluminum, the data of which are from Ref.[26] and not JC data. Results (not shown here) are not as good as those for silver. Therefore, due to silver's quite low intrinsic loss, silver is supposed to be the best option for metal inclusions of metallodielectric MMs to achieve negative index in the visible regime.
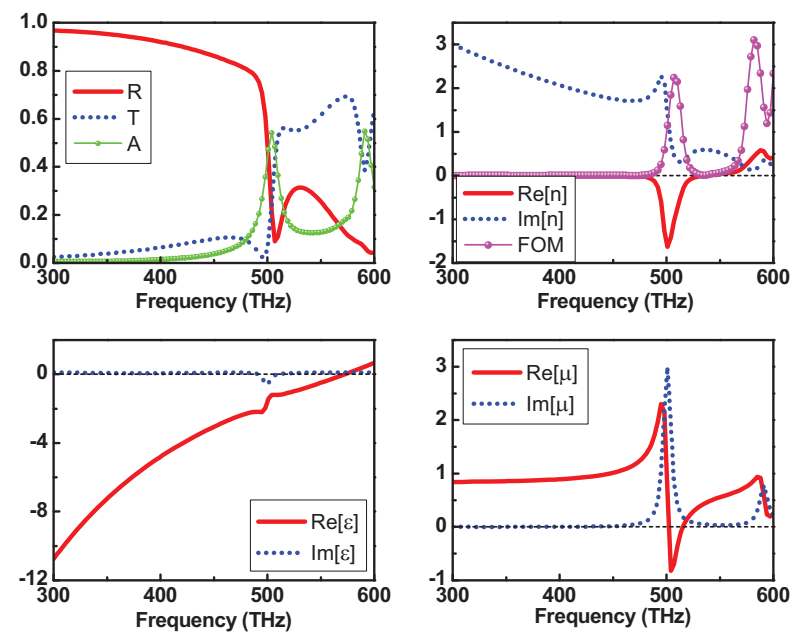

FIG. 7: (Color online) Simulated reflection, transmission, absorption, and retrieved electromagnetic parameters for structure 2, where silver metal is applied with the corresponding JC data.

In conclusion, when MMs go to the optical regime, metallic inclusions begin to play a crucial role to determine the performance of MMs. Compared to various Drude models, which may significantly deviate from the realistic property of the metal for the visible wavelengths, experimentally-measured JC data will be a better choice to model metals to provide accurate predictions for MMs' behaviors. Also, we determined silver, which has a low intrinsic loss, is the best choice for metallic inclusions of optical MMs for achieving negative refractive index.

Work at Ames Laboratory was supported by the Department of Energy (Basic Energy Sciences) under contract No. DE-AC02-07CH11358. This was partially supported by the European Community Project NIM_NIL, Contract No. 228637. 
* nhshen@ameslab.gov

$\dagger$ soukoulis@ameslab.gov

1 V. M. Shalaev, Nature Photon. 1, 41 (2007).

2 C. M. Soukoulis, S. Linden, and M. Wegener, Science $\mathbf{3 1 5}$, 47 (2007).

3 N. I. Zheludev, Science 328, 528 (2010).

4 A. Boltasseva and H. A. Atwater, Science 331, 290 (2011).

${ }^{5}$ C. M. Soukoulis and M. Wegener, Nature Photon. 5, 523 (2011).

${ }^{6}$ R. A. Shelby, D. R. Smith, and S. Schultz, Science 292, 77 (2001).

7 J. B. Pendry, Phys. Rev. Lett. 85, 3966 (2000).

8 Z. Jacob, L. V. Alekseyev, and E. Narimanov, Opt. Express 14, 8247 (2006).

9 Z. Liu, H. Lee, Y. Xiong, C. Sun, and X. Zhang, Science 315, 1686 (2007).

10 I. I. Smolyaninov, Y. J. Hung, and C. C. Davis, Science 315, 1699 (2007).

11 J. B. Pendry, D. Schurig, and D. R. Smith, Science 312, 1780 (2006).

12 D. Schurig, J. J. Mock, B. J. Justice, S. A. Cummer, J. B. Pendry, A. F. Starr, and D. R. Smith, Science 314, 977 (2006).

13 W. Cai, U. K. Chettiar, A. V. Kildishev, and V. M. Shalaev, Nature Photon. 1, 224 (2007).

14 J. Valentine, J. Li, T. Zentgraf, G. Bartal, and X. Zhang, Nature Mater. 8, 568 (2009).

15 L. H. Gabrielli, J. Cardenas, C. B. Poitras, and M. Lipson, Nature Photon. 3, 461 (2009).

16 J. Valentine, S. Zhang, T. Zentgraf, E. Ulin-Avila, D. A. Genov, G. Bartal, and X. Zhang, Nature 455, 376 (2008).

17 C. Garcia-Meca, J. Hurtado, J. Marti, A. Martinez, W. Dickson, and A. V. Zayats, Phys. Rev. Lett. 106, 067402 (2011).

18 D. Chanda, K. Shigeta, S. Gupta, T. Cain, A. Carlson, A. Mihi, A. J. Baca, G. R. Bogart, P. Braun, and J. A. Rogers, Nature Technol. 6, 402 (2011).

19 K. Aydin, I. Bulu, K. Guven, M. Kafesaki, C. M. Soukoulis, and E. Ozbay, New J. Phys. 7, 168 (2005).

20 J. Zhou, Th. Koschny, M. Kafesaki, E. N. Economou, J. B. Pendry, and C. M. Soukoulis, Phys. Rev. Lett. 95, 223902 (2005).

21 S. Xiao, V. P. Drachev, A. V. Kildishev, X. Ni, U. K. Chettiar, H.-K. Yuan, and V. M. Shalaev, Nature 466, 735 (2010).

${ }^{22}$ C. M. Soukoulis and M. Wegener, Science 330, 1633 (2010).

23 J. Zhou, Th. Koschny, M. Kafesaki, and C. M. Soukoulis, Phys. Rev. B 80, 035109 (2009).

24 D. O. Guney, Th. Koschny, and C. M. Soukoulis, Phys. Rev. B 80, 125129 (2009).

${ }^{25}$ P. B. Johnson and R. W. Christy, Phys. Rev. B 6, 4370 (1972).

26 M. A. Ordal, R. J. Bell, R. W. Alexander, Jr., L. L. Long, and M. R. Querry, Appl. Opt. 26, 744 (1987).

27 S. Linden, C. Enkrich, M. Wegener, J. Zhou, Th. Koschny, and C. M. Soukoulis, Science 306, 1351 (2004).

28 We use the software package CST Microwave Studio (Computer simulation Technology GmbH, Darmstadt, Germany).

29 D. R. Smith, S. Schultz, P. Markos, and C. M. Soukoulis,
Phys. Rev. B 65, 195104 (2002).

30 X. Chen, T. M. Grzegorczyk, B.-I. Wu, J. Pacheco, and J. A. Kong, Phys. Rev. E 70, 016608 (2004).

31 D. R. Smith, D. C. Vier, Th. Koschny, and C. M. Soukoulis, Phys. Rev. E 71, 036617 (2005).

32 Th. Koschny, P. Markos, E. N. Economou, D. R. Smith, D. C. Vier, and C. M. Soukoulis, Phys. Rev. B 71, 245105 (2005).

33 Z. Ku, J. Zhang, and S. R. J. Brueck, Opt. Express 17, 6782 (2009).

34 C. Menzel, Th. Paul, C. Rockstuhl, Th. Pertsch, S. Tretyakov, and F. Lederer, Phys. Rev. B 81, 035320 (2010).

35 Some literature adopt a Drude-Lorentz model to fit JC data, for example: V. P. Drachev, U. K. Chettiar, A. V. Kildishev, H.-K. Yuan, W. Cai, and V. M. Shalaev, Opt. Express 16, 1186 (2008). 